\title{
Serological diagnosis of infections sustained by Toxoplasma gondii, Rubella virus and Citomegalovirus, a comparison between two methods
}

\author{
Marco Moretti, Barbara Pieretti, Gabriele Canucoli, Ernesto Delprete \\ Laboratorio di Patologia Clinica Zona Territoriale N ${ }^{\circ}$, Fano (PU) \\ ASUR Marche
}

Key Words: Toxoplasmosis, Rubella, Cytomegalovirus, maternal-fetal infection, immunoassay comparison.

Diagnostica sierologica delle infezioni da T. gondii: confronto fra due metodi

\section{SUMMARY}

The serological diagnosis of infections from Toxoplasma gondii, Rubella virus and Cytomegalovirus is of a great importance especially during pregnancy for the risk of maternal-fetal infections. Serological testing have evolved with time and are many on the market with different levels of specificity and sensivity.

The aim of the study was to determine the analytical and clinical concordance between two methods for the determination of specific IgG and IgM antibodies against T. gondii, Rubella virus and Cytomegalovirus.

The total numbers of samples evaluated for T. gondii, Rubella virus and Cytomegalovirus IgG and IgM were I24 and I27, 83 and 87, I29 and 103, respectively. The comparison methods used for this study were Diamedix ${ }^{\circledR}$ enzime imunoassay run in MAGO-Plus Delta Biologicals $\AA$ Immunodiagnostics System (routinely used) and Vitros@ Eci chemiluminescence immunoassay run in Vitros ECiQ Immunodiagnostics System ORTHO-Clinical Diagnostics Johnson \& Johnson company.Vidas bioMérieux was take as referee method to evaluate discrepant results. Analytical concordance ranged between 87 and $97 \%$, clinical concordance ranged between 93 and $99 \%$.

Despite differences in detection antibodies (specific $\lg G$ and $\lg M$ ) and revelation of signal the methods evaluated show generally good levels of clinical concordance. The results are consistent with references and External Quality Assurance Programs.

La diagnostica delle infezioni da Toxoplasma gondii (T.gondii), virus della Rosolia e Citomegalovirus (CMV) è una attività particolarmente delicata, densa di aspettative e ricadute che richiede specifiche competenze e adeguate risorse tecnologiche $(2,23,27)$. Le infezioni sostenute da questi microorganismi, oltre alla rilevanza in particolari contesti clinici del paziente adulto, sono particolarmente temibili per il rischio di trasmissione materno fetale e la possibilità di produrre danni più o meno gravi al prodotto del concepimento $(18,19,23)$. Per questo è necessario predisporre un appropriato percorso preventivo, diagnostico e terapeutico multidisciplinare e multiprofessionale $(16,17,26,28)$.

Dal punto di vista diagnostico il Legislatore ha previsto con il Decreto Ministeriale 10 settembre 1998 "Protocolli di accesso agli esami di laboratorio e di diagnostica strumentale per le donne in gravidanza e a tutela della maternità" l'esecuzione di esami preconcezionali e pre-natali al fine di individuare le infezioni contratte in gravidanza e contrastare le loro possibili complicanze (4).

Il compito del Microbiologo Clinico è quello di predisporre un percorso diagnostico, spesso in rete, che si avvale di metodi integrati opportunamente scelti e applicati per fornire un'appropriata informazione diagnostica e una valida consulenza nell'interpretazione dei risultati (10).

La diagnostica delle infezioni sostenute da T. gondii, virus della Rosolia e CMV si basa sulla ricerca sierologica delle immunoglobuline di classe IgG e IgM specifiche utilizzando metodi che devono garantire alta sensibilità e specificità (20) il cui goal è definire lo stato immunitario del soggetto (1). Esistono diversi metodi di determinazione delle $\operatorname{IgG}$ e $\operatorname{IgM}$ specifiche che differiscono sia per come avviene il riconoscimento dell'analita (in base agli antigeni di cattura utilizzati) sia per la tecnologia utilizzata nel rilevare il segnale (colorimetria, chemiluminescenza, fluorescenza, immunofluorescenza indiretta).

Il nostro lavoro si è proposto di verificare i tassi di concordanza analitica e clinica nella determinazione delle IgG e IgM specifiche prodotte verso T. gondii, virus della Rosolia e CMV. Il confronto è stato condotto fra il metodo immunoen- zimatico su micropiastra Diamedix ${ }^{\circledR}$ (Diamedix, Miami, USA), in uso da otto anni nel nostro laboratorio, e il metodo immunometrico con rilevazione in chemiluminescenza potenziata Vitros ${ }^{\circ}$ Eci (ORTHO-Clinical Immunodiagnostics System Johnson \& Johnson company, USA).

Lo studio è stato condotto analizzando 321 campioni provenienti dalla nostra routine arricchendo la casistica con alcuni campioni conservati in sieroteca a $-80^{\circ}$. I campioni da sieroteca sono stati aggiunti perché caratterizzati dalla presenza di IgM specifiche (13 campioni positivi per IgM anti-T. gondii, 10 campioni positivi per IgM anti virus della Rosolia e 6 campioni positivi per IgM anti CMV) o da possibili interferenti (19 campioni con IgM anti virus di Epstein-Barr) al metodo in uso.

I campioni di siero sono stati preparati centrifugando a 3500 rpm il sangue venoso periferico raccolto, attendendo almeno 30 minuti, in provette in polipropilene Vacuette ${ }^{\circledR}$ Z Serum Sep. Clot Activator Greiner Bio-one da $7 \mathrm{ml}$. I campioni provenienti da sieroteca a $-80^{\circ}$ sono stati scongelati, centrifugati e analizzati su entrambe le strumentazioni nella stessa giornata. I campioni sono stati analizzati con il metodo Diamedix ${ }^{\circledR}$, eseguito su strumentazione automatizzata MAGO-Plus Delta Biologicals ${ }^{\circledR}$ (metodo in uso) ed il metodo Vitros $(\mathrm{C}$ Eci su strumentazione automatizzata random-access Vitros $(\mathrm{E}) \mathrm{EciQ}$ ORTHO-Clinical Diagnostics. Durante la sperimentazione i metodi sono stati sottoposti a controllo interno di qualità per tutti i dosaggi valutati utilizzando materiali di controllo specifici analizzati quotidianamente su due livelli. La precisione analitica di entrambi i metodi e per tutti i dosaggi valutati è risultata conforme alle regole di accettabilità del controllo interno da noi utilizzate.

Il metodo Diamedix ${ }^{\circledR}$ determina le IgG specifiche con metodo immunoenzimatico su micropiastra (Enzyme Linked ImmunoSorbent Assay: ELISA). Il siero del paziente è dispensato nella cuvetta di reazione coattata con l'antigene (antigeni di T. gondii, antigeni inattivati e purificati del virus della Rosolia e antigeni di CMV parzialmente purificati e inattivati con beta-propriolattone). Dopo una prima incubazione di 30 minuti un lavaggio elimina il non legato. In segui-

\section{Corresponding author: Marco Moretti}

Laboratorio Analisi Chimico-Cliniche e Microbiologiche Ospedale S. Croce

Zona Territoriale 3 Fano (PU) - Viale Vittorio Veneto 261032 Fano (PU) - Tel. 072I-882695 Fax 072I-882304

E-mail: marcomoretti I8@libero.it 
to è aggiunto un coniugato costituito da anticorpi monoclonali anti-IgG umane legati con perossidasi. L'aggiunta di 3.3', 5.5 ' - tetrametil-benzidina (TMB) che funge da substrato comporta una reazione colorimetrica che viene bloccata mediante l'aggiunta di una soluzione di acido solforico $0.3 \mathrm{M}$. I valori di assorbanza letta a 450 nanometri $(\mathrm{nm})$ sono confrontati con la curva di calibrazione a sei punti eseguita in ogni seduta analitica. L'utilizzo di calibratori riferibili al World Health Organization (WHO) 3rd International Standard Preparation 1997 per T. gondii, 1st International Standard Preparation WHO per il virus della Rosolia e Proposed International Standard WHO per CMV permette di esprimere i risultati in Unità Internazionali/ millilitro (U.I./ml).

La Tabella 1 riporta i valori di concentrazione delle $\operatorname{IgG}$ specifiche che discriminano i campioni

in negativi, indeterminati e positivi (5).

La determinazione delle IgM specifiche avviene previa cattu-

ra di questa classe di immunoglobuline mediante anticorpi anti-IgM umane fissati al pozzetto di reazione e successiva rivelazione delle IgM specifiche sfruttando la loro capacità di legare l'antigene (tachizoiti purificati, inattivati e sonicati di T. gondii, virus della Rosolia inattivato e purificato e CMV parzialmente purificato e inattivato con beta-propriolattone). Anche in questo test la rilevazione del segnale è ottenuta sfruttando l'azione della perossidasi sul TMB con lettura dell'assorbanza a $450 \mathrm{~nm}$. I valori di assorbanza ottenuti sono confrontati ed espressi come rapporto con quella prodotta da un controllo interno (cut-off) analizzato in triplicato. La Tabella 1 riporta i valori del rapporto fra la densità ottica del campione e del cut-off che discriminano i risultati in positivi, negativi o indeterminati (6).

I dosaggi Vitros $\subseteq$ Eci sono metodi immunometrici con rilevazione in chemiluminescenza potenziata.

La determinazione delle IgG specifiche del campione si basa sulla reazione fra queste e gli antigeni (antigeni di T. gondii, virus della Rosolia, e CMV) con cui sono sensibilizzati i pozzetti di reazione. Dopo incubazione e lavaggio sono aggiunti anticorpi monoclonali anti-IgG umane coniugati con perossidasi di rafano (HRP). Seguono una nuova fase di incubazione, lavaggio e l'aggiunta di substrati luminogeni (un derivato del luminolo e un sale peracido) ed un agente di trasferimento di elettroni (acetanilide sostituita). La perossidasi di rafano catalizza l'ossidazione del derivato del luminolo dando origine a produzione di luce. L'agente di trasferimento di elettroni provoca un aumento del livello di luce prodotta e ne prolunga l'emissione. I segnali luminosi, prodotti con intensità direttamente proporzionale alla concentrazione di IgG specifiche presenti nel campione, sono rilevati da un luminometro. I valori misurati sono confrontati con quelli prodotti dalla relativa curva di calibrazione riferibile alle standardizzazioni precedentemente citate $(12,29)$ permettendo così di esprimere i risultati in U.I./ml.

La Tabella 1 riporta i valori di concentrazione delle IgG specifiche che discriminano i campioni

in negativi, indeterminati e positivi per $\mathrm{i}$ dosaggi Vitros $\subset$ Eci.

La determinazione delle IgM specifiche utilizza un metodo a cattura. Previa diluizione del campione le IgM del campione reagiscono inizialmente con anticorpi monoclonali anti-IgM umane di topo biotinilati. L'immunocomplesso è catturato nei pozzetti coattati con streptavidina mentre il materiale non legato è rimosso mediante lavaggio. Un secondo anticorpo monoclonale legato con 1'antigene specifico e marcato con perossidasi di rafano (HRP) viene quindi cattu-

Tabella 3. Dati complessivi di concordanza analitica e clinica ottenuti per i singoli anticorpi.

\begin{tabular}{lccc}
\hline & & \multicolumn{2}{c}{ Concordanza } \\
\hline Classe Ig & N. Campioni & Analitica & Clinica \\
\hline Toxoplasma IgG & 124 & $89 \%$ & $95 \%$ \\
\hline Toxoplasma IgM & 127 & $93 \%$ & $98 \%$ \\
\hline Rosolia IgG & 83 & $87 \%$ & $93 \%$ \\
\hline Rosolia IgM & 87 & $95 \%$ & $98 \%$ \\
\hline Citomegalovirus IgG & 129 & $97 \%$ & $97 \%$ \\
\hline Citomegalovirus IgM & 103 & $94 \%$ & $99 \%$ \\
\hline
\end{tabular}

rato dall'IgM specifica eventualmente legata ai pozzetti. Un secondo lavaggio rimuove il non legato. Come per le IgG specifiche la rilevazione avviene tramite chemiluminescenza potenziata utilizzando l'azione di HRP sui substrati luminogeni con produzione di luce proporzionale al contenuto delle IgM specifiche presenti nel campione $(12,30)$. I risultati sono espressi come rapporto rispetto ad un cut-off interno.

La Tabella 1 riporta i valori di riferimento per le IgM specifiche per il metodo Vitros $₫$ Eci espresse come rapporto fra segnale e cut-off. 
Tutti i campioni sono stati analizzati con entrambe le metodiche, ogni singolo risultato è stato considerato negativo, indeterminato o positivo rispetto ai valori di riferimento indicati dai produttori e riportati nella Tabella 1 .

In seguito sono stati raccolti, appaiati e confrontati su foglio di calcolo elettronico Microsoft Office Excel@ i risultati ottenuti con i due metodi.

Il confronto ha permesso di esprimere per ogni coppia di risultati una condizione di concordanza (negativo/negativo; positivo/positivo; indeterminato/indeterminato) o discordanza analitica (negativo/indeterminato, negativo/positivo, indeterminato/positivo e viceversa). La concordanza analitica complessiva è espressa come rapporto fra dati analiticamente concordanti e totale dei confronti eseguiti per quel determinato dosaggio. Le coppie di risultati analiticamente discordanti sono state sottoposte a verifica per valutare la possibilità di generare o meno concordanza clinica.

Questa esprime la possibilità che valori analiticamente discordanti possano tradursi clinicamente in comportamenti equivalenti. Una delle situazioni più frequenti è quella di campioni con IgG specifiche indeterminate ad un metodo e negative all'altro. In questo caso la discordanza analitica si traduce in concordanza clinica considerando in entrambi i casi il soggetto come potenzialmente recettivo e quindi proponendo da un punto di vista clinico un controllo nel tempo o nel caso del virus della Rosolia una eventuale vaccinazione.

In altre situazioni la discordanza analitica è stata sottoposta all'analisi del campione con un terzo metodo che ha svolto funzioni di "arbitro". Nella nostra attività utilizziamo come supporto diagnostico al metodo Diamedix ${ }^{\circledR}$ per la determinazione delle IgG e IgM specifiche e per l'esecuzione dei test di avidità per le $\mathrm{IgG}$ specifiche il metodo immunometrico con rivelazione in fluorescenza (ELFA) Vidas ${ }^{\circledR}$ (bioMérieux ${ }^{\circledR}$ Marcy l'Etoile, France).

Questo ha permesso di ind e alcune situazioni di discordanza analitica quali valori indeterminati di IgM con un metodo e negativi/positivi all'altro.

La concordanza clinica complessiva è espressa come rapporto fra comportamenti clinici concordanti e totale dei confronti eseguiti per quel dosaggio.

Si riporta nella Tabella 2 i dati analitici ottenuti per i singoli test per le due metodiche confrontate. La Tabella 3 riporta $i$ dati complessivi di concordanza analitica e clinica ottenuti per i singoli anticorpi.

Il confronto per le IgG specifiche evidenzia valori di concordanza analitica dell'89\% (110 campioni su 124 complessivi) per T. gondii, $87 \%$ per Rosolia (72 campioni su 83 complessivi) e 97\% per CMV (124 campioni su 129 complessivi). La concordanza clinica cresce rispettivamente al 95\%, $93 \%$ e 97\%. Questo perché i campioni indeterminati ad un metodo e negativi all'altro comportano un potenziale stato recettivo che induce comportamenti clinici equivalenti (monitoraggio nel tempo, eventuale vaccinazione). Per i campioni che rimagono discordanti in alcuni casi (7 positivi per Toxoplasma IgG per Diamedix $₫$ e negativi su Vitros $\bigodot$ Eci, 6 indeterminati per IgG anti Rosolia per Diamedix ${ }^{\circledR}$ e positivi per Vitros@ Eci) l'analisi dettagliata ha evidenziato valori prossimi ai limiti analitici di discriminazione dell'interpretazione del risultato. Nel caso dei campioni discordanti per le IgG anti Citomegalovirus i 5 risultati valutati su Vidas ${ }^{\circledR}$ (bioMérieux ${ }^{\circledR}$ ), come metodo "arbitro", hanno condotto a concordanze alternate e non univoche con i due metodi confrontati.

L'analisi della concordanza analitica per le IgM ha prodotto valori pari al 93\% (118/127 campioni) per T. gondii, 95\% (82/87 campioni) per Rosolia e 94\% (97/103 campioni) per CMV. Questi valori aumentano come concordanza clinica a valori di $98 \%, 98 \%$ e $99 \%$ rispettivamente considerando i casi di IgM indeterminate.

In questi casi l'utilizzo del metodo "arbitro" ha confermato la negatività per i 6 campioni indeterminati/negativi per Toxoplasma IgM, del campione indeterminato/negativo per IgM anti virus della rosolia e per i 5 campioni negativi/inde- terminati per IgM anti Citomegalovirus.

Rimangono invece alcuni campioni ( 2 campioni per IgM anti Toxoplasma, 2 campioni per IgM anti virus della Rosolia e un campione per IgM anti Citomegalovirus) discordanti.

L'analisi di questi campioni con metodo "arbitro" ha anche in questo caso evidenziato valori alternati e non univoci di concordanza con i due metodi confrontati.

I campioni positivi per IgM anti-virus di Epstein-Barr al metodo in uso non hanno evidenziato reazioni crociate nella determinazione delle IgM specifiche oggetto della valutazione sui due sistemi analitici.

I metodi applicati per la definizione dello stato immunitario verso T. gondii, virus della Rosolia e CMV devono essere altamente specifici e sensibili in modo da ridurre al massimo l'incidenza di risultati falsamente positivi e soprattutto falsamente negativi $(1,2)$.

Queste caratteristiche differiscono fra i vari metodi in commercio che presentano notevoli differenze sia in fase di riconoscimento dell'analita sia in fase di rilevazione del segnale. In particolare per il riconoscimento dell'analita i diversi metodi impiegano preparazioni differenti di antigeni. Le preparazioni variano da miscele antigeniche complesse prodotte su colture in animale ( $T$. gondii) o cellulari (virus della Rosolia e CMV) ad antigeni ricombinanti (18) mentre per la rilevazione del segnale sono utilizzate diverse tecnologie (colorimetria, chemiluminescenza, fluorescenza, immunofluorescenza indiretta).

I due metodi confrontati differiscono sia per come avviene il riconoscimento dell'analita sia per la tecnologia di rilevazione del segnale (colorimetria vs chemiluminescenza).

$\mathrm{Si}$ sottolinea come entrambe le metodiche nella determinazione delle IgM specifiche utilizzino invece sistemi "di cattura" che, come dimostrato in letteratura (11), garantiscono prestazioni analitiche migliori riducendo soprattutto l'azione di eventuali interferenti.

Il confronto, anche se ha riguardato una popolazione campionaria numericamente limitata è stato condotto su una casistica volutamente stressante ottenuta arricchendola con campioni positivi per IgM specifiche al metodo in uso e campioni con possibili interferenze (campioni positivi per IgM anti virus di Epstein-Barr).

I livelli di concordanza analitica ottenuta oscillano per le IgG specifiche fra l' $87 \%$ e il $97 \%$ e il $93 \%$ e il $95 \%$ per le IgM. I valori di concordanza clinica aumentano oscillando fra il 93\% e il $97 \%$ per le $\operatorname{IgG}$ e il 98 e il $99 \%$ per le IgM.

Questi risultati indicano che esistono per le IgG specifiche, nonostante la presenza di preparazioni standard internazionali di riferimento che dovrebbero facilitare la comparazione fra metodi diversi, delle difficoltà nell'armonizzazione dei risultati soprattutto in corrispondenza dei valori prossimi ai limiti analitici di discriminazione dell'interpretazione del risultato $(9,31)$. Anche l'utilizzo di un metodo "arbitro" non comporta una chiarificazione del quadro avendo sui campioni testati concordanze alternate e non univoche con $\mathrm{i}$ due metodi confrontati (20).

Come dimostrato in letteratura (7), tutti i metodi presenti sul mercato hanno una limitata capacità di separare $i$ campioni positivi e negativi per le $\operatorname{IgG}$ specifiche, esiste quindi il rischio che solo per motivi attribuibili alla variabilità analitica un campione in corrispondenza dei limiti interpretativi del risultato possa essere considerato negativo o positivo. Nonostante il processo internazionale di standardizzazione abbia migliorato la situazione di concordanza nella determinazione delle IgG specifiche (14) rispetto alla situazione precedente (8), rimangono ancora serie problematiche nell'intercambiabilità e nel grado di correlazione e concordanza dei risultati ottenuti con differenti piattaforme analitiche $(7,20)$. Per questo si impongono ulteriori sforzi e miglioramenti nel processo di standardizzazione (7).

Per le IgM specifiche si sono ottenuti alti livelli di concordanza. Rimangono comunque alcuni campioni (2 campioni per IgM anti Toxoplasma, 2 campioni per IgM anti virus della 
Rosolia e un campione per IgM anti Citomegalovirus) discordanti.

L'analisi di questi campioni con metodo "arbitro" ha anche in questo caso evidenziato valori alternati e non univoci di concordanza con i due metodi confrontati.

Anche la letteratura recente descrive tali difficoltà diagnostiche $(3,14,18,20,25,31)$ con risultati di concordanza estremamente variabili a seconda delle caratteristiche della popolazione campionaria e a seconda dei metodi confrontati sottolineando ancora la possibilità di avere risultati falsamente negativi o falsamente positivi soprattutto per campioni con bassi livelli di IgM specifiche $(13,15,22,24,32)$.

Questi risultati sono in linea con la fotografia dello "stato dell'arte" che ci forniscono i programmi di Verifica Esterna di Qualità (VEQ) quali il Programma di VEQ organizzato dal Centro Regionale di Riferimento Sicurezza e Qualità in Laboratorio Azienda Ospedaliera Universitaria Careggi Firenze al quale il nostro laboratorio istituzionalmente partecipa (21). In definitiva le diverse architetture dei metodi disponibili comportano una percentuale più o meno contenuta, ma non eliminabile, di campioni con risultati discordanti che possono condurre a comportamenti clinici non equivalenti.

Il confronto, confermando letteratura e "stato dell'arte", dimostra che i due metodi si comportano come sistemi sostanzialmente equivalenti se applicati su larga scala con la possibilità di avere alternativamente una migliore definizione analitica assolutamente specifica sul singolo campione e non prevedibile a priori.

$\mathrm{Ne}$ deriva che, non esistendo al momento un metodo analitico infallibile e di riferimento per questa specifica attività diagnostica, il Microbiologo Clinico e la Direzione del Laboratorio devono necessariamente conoscere e far conoscere i limiti dei sistemi, predisporre metodi integrati di approfondimento e conferma, declinare le prestazioni analitiche nella organizzazione del flusso operativo e correlare sul singolo campione i risultati alle informazioni ed alle motivazioni cliniche alla base della richiesta analitica.

\section{BIBLIOGRAFIA}

1. Antico A, Bacelle L. Governo Clinico dei test di laboratorio per la diagnosi sierologica di infezione da Toxoplasma gondii e Citomegalovirus. Convegno Regionale SIMeL Veneto 27/04/2005 Battaglia Terme (PD).

2. Best JM, O' Shea G, et al. Interpretation of rubella serology in pregnancy: pitfalls and problems. Brit Med J 2002; 325: 147-48.

3. Decoster A, Lambert N, Germaneau C, Masson C. Toxoplasmosis sierodiagnosis: comparison of Access Toxo IgM II assay compared to Axsym Toxo IgM and Vidas toxo IgM assays. Ann Biol Clin 2000; 58:721-27.

4. Decreto Ministeriale, Ministero della Sanità 10 settembre 1998 "Protocolli di accesso agli esami di laboratorio e di diagnostica strumentale per le donne in gravidanza e a tutela della maternità". G.U. n 245, 20 Ottobre 1998

5. Diamedix ${ }^{\circledR}$ Toxoplasma, Rubella, CMV IgG Enzime Immunoassay test.

6. Diamedix ${ }^{\circledR}$ Toxoplasma, Rubella, CMV IgM Capture Enzime Immunoassay test.

7. Dimech W, Panagiotopoulos L, et al. Evaluation of eight anti-Rubella Virus immunoglobulin G immunoassays that report results in International Units per millilitre. J Clin Microbiol 2008; 46:1955-60.
8. Dimech W, Bettoli A, et al. Multicenter evaluation of five commercial rubella virus immunoglobulin $\mathrm{G}$ kits which report results in International Units per millilitre. J Clin Microbiol 1992; 30: 633-41.

9. Flori P, Chene G, Varlet MN, Sung RT. Toxoplasma gondii in pregnant woman: characteristics and pitfalls. Ann Biol Clin 2009; 2: 125-33.

10. Gruppo di Lavoro AMCLI "Infezioni Materno-Fetali" Attualità in tema di infezioni materno-fetali. Editing Berrino Printer San Mauro T se (TO), 2009.

11. Hudson P, Morgan Capner P. Evaluation of 15 commercial enzyme immunoassays for the detection of rubella-specific IgM. Clin Diagn Virol 1996; 5: 21-6.

12. Kasper DC, et al. Evaluation of the VitrosECiQ Immunodiagnostics System for detection of anti Toxoplasma immunoglobulin $\mathrm{G}$ and immunoglobulin $\mathrm{M}$ antibodies for confirmatory testing for acute Toxoplasma gondii infection in pregnant women. J Clin Microbiol 2009; 47: 164-67.

13. Lazzarotto T, Galli C, et al. Evaluation of Abbott AxSYM citomegalovirus (CMV) immunoglobulin M (IgM) assay in conjuction with other tests and CMV IgG avidity assay. Clin Diagn Lab Immunol 2001; 8: 196-98.

14. Maudry A, Chene G, et al. Bicentric evaluation of six anti-toxoplasma immunoglobulin $\mathrm{G}(\operatorname{IgG})$ automated immunoassayand comparison to the Toxo II IgG Western blot. Clin Vaccine Immunol 2009; 16: 1322-6.

15. Meek B, van Gool T, Gilis H, Peek R. Dissecting the IgM antibody response during the acute and latent phase of toxoplasmosis. Diagn microbial Infec Dis 2001; 41: 131-9.

16. Montoya JG, Liesenfeld O, Kinney S, Press C, Remington JS. Vidas test for avidity of Toxoplasma specific immunoglobulin $\mathrm{G}$ for confirmatory testing of pregnant women. J Clin Microbiol 2002; 40: 2504-08.

17. Moretti M, Pieretti B, et al. Sieroepidemiologia delle infezioni daToxoplasma gondii, virus della Rosolia e Citomegalovirus in una coorte di gravide. Microbiologia Medica 2006; 21: 121-26.

18. Owen WE, Martins TB, Litwin CM, Roberts WL. Performance Characteristics of six Immulite 2000 TORCH assays. Am J Clin Pathol 2006; 126: 900-05.

19. Petersen E. Toxoplasmosis. Semin fetal Neonatal Med 2007; 12: 214-23.

20. Petersen E, BorobioMV, et al.European multicentric study of the Liason automated diagnostic system for determination of Toxoplasma gondii specific immunoglobulin G (IgG) and IgM and the IgG avidity index. J Clin Microbiol 2005; 43: 1570-74.

21. Programma di Valutazione Esterna di Qualità. Centro Regionale di Riferimento S.OD Sicurezza e Qualità in Laboratorio Azienda Ospedaliera Universitaria Careggi Firenze.

22. Revello MG, Gorini G, Gerna G. Clinical evaluation of chemiluminescence immunoassay for determination of immunoglobulin $\mathrm{G}$ avidity to human Citomegalovirus. Clin Diagn Lab Immunol 2004; 11: 801-04.

23. Revello MG, Gerna G. Diagnosis and management of human cytomegalovirus infection in the mother, fetus and newborn infants. Clin Microbiol Rev 2002; 15: 680-715.

24. Robert A, Hedman K, et al. Multicenter evaluation of strategies for serodiagnosis of primary infection with Toxoplasma gondii. Eur J Clin Microbiol Infect Dis 2001; 20: 467-74.

25. Robert-Gangneux F, Bourhis C, Chevrier S, Gangneux JP. Evaluation of DPC Immulite 2000 Toxoplasma quantitative IgG/IgM kits for automated toxoplasmosis serology with Immulite 2000. J Clin Lab Anal 2009; 23: 336-40.

26. Schinella M, Turci V. La sierologia del complesso TORCH. Applicazioni ed Interpretazioni. Med Lab 1998; 2: 156-9.

27. Sensini A. Toxoplasma gondii infection in pregnancy: opportunities and pitfalls of serological diagnosis. Clin Microbiol Infect 2006; 12: 504-12.

28. Tamer GS, Dundar D, Caliskan E. Seroprevalence of Toxoplasma gondii, rubella and cytomegalovirus among pregnant women in western region of Turkey. Clin Invest Med 2009; 32: E43-7.

29. Vitros $\mathbb{C}$ Eci Toxoplasma, Rubella, Cytomegalovirus IgG assay.

30. Vitros $\mathbb{C}$ Eci Toxoplasma, Rubella, Cytomegalovirus IgM assay.

31. Vlaspolder F, Singer P, Smit A, Diepersloot RJ. Comparison of Immulite with Vidas for detection of infection in a low prevalence population of pregnant women in The Netherlands. Clin Diagn Lab Immunol 2001; 8: $552-55$

32. Wilson M, Remington JS, et al. Evaluation of six commercial kits for detection of human immunoglobulin $\mathrm{M}$ antibodies to Toxoplasma gondii. J Clin Microbiol 1997; 35: 3112-15. 